\title{
Unmanned driving research of underground scraper based on laser measurement system
}

\author{
Jianguo $\mathrm{Li}^{1,2}$ a , Kai Zhan ${ }^{2, \mathrm{~b}}$, Xin $\mathrm{GuO}^{2, \mathrm{c}}$ and Hengtong $\mathrm{Li}^{2, \mathrm{~d}}$, \\ ${ }^{1}$ University of Science and Technology Beijing, Beijing, 100083, China \\ ${ }^{2}$ Beijing General Research Institute of Mining and Metallurgy, Beijing, 100160,China \\ ali_jg@bgrimm.com, bhan_k@bgrimm.com, 'guo_x@bgrimm.com, ${ }^{d}$ lihengtong1994@163.com
}

Keywords: scraper, deviation, navigation, unmanned.

Abstract. Combined with the special working environment of underground tunnels, the method of unmanned driving technique for underground scraper based on laser measurement system is proposed. The key factor is obtaining the horizontal position deviation which is used to realize the tendency to zero by adjusting fore and post splice angle of scraper through real-time control. How to calculate the deviation by laser measurement system mounted on the scraper and the controlling algorithm for unmanned driving are introduced. Unmanned driving tests using the algorithm combined with practical application are carried out on a two-cubic scraper in the underground tunnel. The result shows that the method can realize unmanned driving of scraper with certain application value.

\section{Introduction}

With the growing of need for metal mineral resource, more and more mines are going to deep exploration. Intelligence unmanned underground equipment has become the development trend [1]. The relevant intelligent technique of the scraper is one of the most important underground trackless equipment. Intelligent underground scraper needs no underground shift of personnel, so the production time is fully ensured, for the efficiency of underground exploration can be improved significantly and on-site staff can be reduced greatly in cooperation with intelligent control technique and advance state monitoring technique, the safety of personnel can be ensured further[2]. On this foundation, this paper proposes a method of unmanned driving technique of underground scraper based on laser measurement system to provide theoretical and technical support to intelligent scraper.

\section{Unmanned driving scraper}

There are many methods to realize the unmanned driving of the scraper in underground tunnel. During these methods, the auto navigation is the key technology which can enable underground scraper to realize unmanned driving [3-5]. Navigation can be divided into absolute navigation and relative navigation under existing technical conditions. Absolute navigation technology refers to the digital information of scraper unmanned driving environment map has been saved in board computer in advance, then the best trajectory can be designed under the digital map coordinate system for scraper to follow rigidly, the core is scraper can perceive the real-time position information of scraper through self-sensor in this way. Relative navigation refers to perceive surrounding infrastructure or local objects through board sensor to realize navigation, it is also known as reactive navigation technology. The typical method of technique based on "walk along the wall" is controlling scraper to move along the centerline of roadway with principle of not touching the wall.

This paper's navigation is similar with the relative navigation. As we know, the centerline of underground tunnels is most the best trace of the scraper while it transports the mine between the load point and dump point [6]. The deviation information is mixed to realize the tendency to zero of deviation by adjusting fore and post splice angle of scraper through real-time control.

\section{Laser measurement system}

For the unmanned scraper, the key factor of controlling is how to identify the surrounding tunnel information which can used to guide the next action. As the known technologies, laser measurement 
system is one of the useful methods to apply for the controlling with many advantages, such as low cost, convenient operation etc. The laser measurement system is a non-contact, stand-alone remote measuring system designed for use in industrial environments [7]. It is usually used for distance measuring and obstacles detecting. The laser measurement system operates by measuring the time of flight of laser light pulses: a pulsed laser beam is emitted and reflected if it meets an object. The reflection is registered by the laser measurement's receiver. The time between transmission and reception of the impulse is directly proportional to the distance between the laser measurement system and the object (time of flight). As an example, one of the kinds of laser measurement system which is applied in this article is shown in Fig. (1).

\section{Method of unmanned driving}

In this paper, the scraper does not need to "know" with any accuracy where it is in the environment with respect to some global co-ordinate frame. It only needs to know where it is relative to the navigation infrastructure or local objects. The method of unmanned driving is keeping the scraper moving on follow the centerline of the underground tunnels. The laser measurement is mounted on top of the cab on the scraper to detect the distance between the tunnel and scraper.

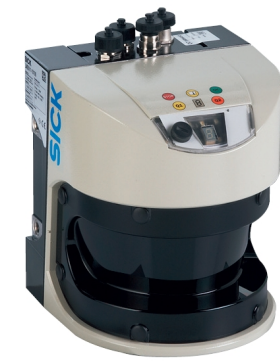

Fig. 1. Laser measurement system

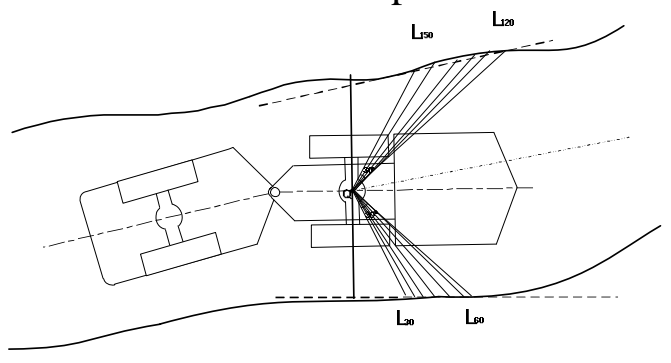

Fig. 2. Laser measurement system on the scraper

From Fig2, the horizontal deviation is the major deviation between the target path and pursuit trail of underground scraper. The design principle of controller is modifying horizontal to 0 constantly. At certain driving speed, the unmanned driving controller is designed into horizontal deviations fusion feedback correction controller, in which the formula of general feedback $e$ is:

$e=\sum_{i=0}^{i=m} \frac{L_{180-i}-L_{i}}{L_{180-i}+L_{i}}$

In which:

$i$ is the scanning beam number in calculation of the side scanning zone.

$L_{i}$ is the measured data of No. $\mathrm{i}$.

$m$ is the scanning beam quantity in calculation of the side scanning zone.

In order to calculate the accurate $e$, the angle of left side scanning is chosen between $30^{\circ}$ and $60^{\circ}$, the right side scanning is chosen between $120^{\circ}$ and $150^{\circ}$ as shown in Fig 2 .

\section{Controlling algorithm design}

While the scraper is under unmanned driving, the turning angle is controllable variable as one suitable value of speed would be taken for normal driving, so the control on trajectory of scraper mainly refers to real-time control on steering angle of scraper. PID control of variable parameter is adapted. Fused deviation $e$ shall be inputted to unmanned driving controller of PID as controllable variable and processed by PID unmanned driving controller with fused deviation $e$ tending to 0 as goal, so as to realize unmanned driving of underground scraper with steering systematical movements controlled by output controlling voltage of the valve.

The controlling algorithm goes like:

The proportional coefficient $K_{p}$, integral coefficient $K_{i}$ and differential coefficient $K_{d}$ are given value, Adapted circle $\mathrm{T}$ is given.

When $k=1$, initialize $e=0$, when $k=2$, initialize $e(k-1)=e(k-2)=0$. 
The unmanned driving of scraper would begin when $\mathrm{k}=2, u(k-1)=u(k-2)=0$.

Obtain the current speed of scraper $V_{g}(k)$

Input the position of initial $\mathrm{X}$ axis direction of scraper with $s(1)=0$.

Calculate the infused feedback value as equation (1).

Calculate output steering controlling voltage $u(1)$ after feedback is processed by PID controller.

$u(k)=u(k-1)+\Delta u(k)$

$u(1)$ in formula (2) is the previous voltage controlled by circle time with $\Delta u(1)$ being the increment for controlling the steering voltage of scraper, while $e(k-1)$ is the fused feedback value of previous sample time, $e(k-2)$ is the fused feedback value of previous sample time of $e(k-1) . K_{p}$ is the proportional coefficient, $K_{i}$ is integral coefficient and $K_{d}$ is the given value of differential coefficient.

For preparation for the next time, calculate the trip distance of scraper

$s(k+1)=s(k)+V_{g}(k) * T$

According to the trip distance $s(2)$, calculate corresponding horizontal displacement deviation.

Iterative storage deviation

$\mathrm{e}(k-1) \longrightarrow e(k-2)$

$e(k) \longrightarrow e(k-1)$

$u(k) \longrightarrow u(k-1)$

Repeat the above procedure to calculate the deviations of scraper at real-time base with scanning laser measurement system to output steering control instruction of scraper, in this way, unmanned driving control of underground scraper can be realized.

\section{Experimental analyses on two-cubic scraper}

To further verify the feasibility of the algorithm, the method shall be applied in the real unmanned driving control of two-cubic diesel scraper. In the actual experiment, the acquirement of hinged joint applies real-time measurement with angle sensor and the acquirement of real-time speed of scraper is drawn from sensor in gearbox with internet bus at real-time base. The map setting of unmanned driving of scraper goes like Fig 3, as one straight line $47000 \mathrm{~mm}$ with a while tracking trail is the central line of roadway. The average width of the tunnel is 3.8 meters and the width of the scraper is 1.8 meters.

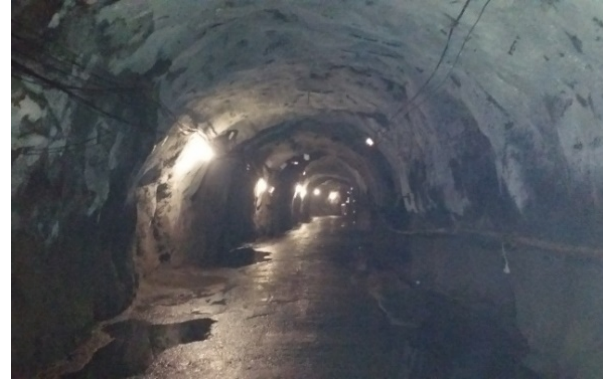

Fig3. Underground test tunnel

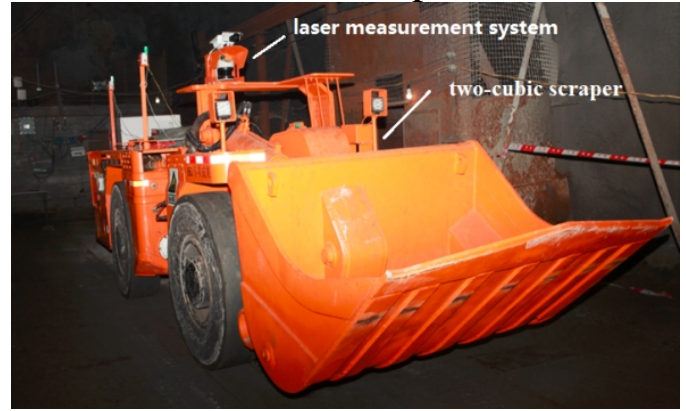

Fig4. The trial two-cubic scraper

In the experiment, the aim of the scraper unmanned controlling is driving under a given underground tunnel environment. The laser measurement system is mounted on top of the cab as shown in Fig4. During the unmanned driving, the laser measurement system detects the front district which can use to calculate the deviations in real time. The key factor of the unmanned driving is keeping the distance from left equal to the right. The velocity of the scraper is set to a fixed value about $1.5 \mathrm{~km} / \mathrm{h}$ while the electronic gear and the electronic pedal are given a fixed value. The laser measurement system can return 181 number data with the angular resolution is $1^{\circ}$ every one scan of $180^{\circ}$ while the rate is $50 \mathrm{HZ}$. The data is processed by a embed computer on the scraper, it can also output analog and digital signal to control the steering and velocity.

For the real experiment, take e as the proportional coefficient of PID $K_{p}=56, K_{d}=0, K_{i}=0.2$. Horizontal position deviation is set to 0 . The real-time changing of turning angle of actual tested tracking trail is showed in the Figure.5. 
Seen from the Figure.5, the algorithm can follow the trail of roadway central line well and the scraper can adjust its posture and speed by adjusting the fore and post hinged angle constantly during the self-driving process, so as to reach the goal of following the planned path. Besides, this system owns certain robustness and stability.

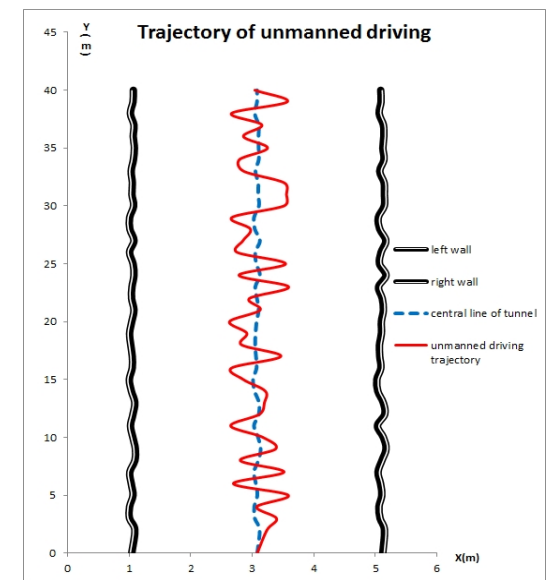

Fig5. Trajectory of unmanned driving of the scraper

\section{Conclusion}

Based on the laser measurement system, this paper designed a controller of unmanned navigation combined with horizontal position deviation navigation. The control algorithm work as overall correction of feedback during the driving process. In this way, unmanned navigation driving of scraper is realized and tested under real environment. The trajectory of unmanned driving during the driving process is analyzed. The experimental result shows that self-navigation control algorithm based on laser measurement system can realize the unmanned driving function of scraper well.

\section{Acknowledgment}

The authors are grateful to the financial supported by the National High Technology Research Program of China ("863" Project)(Grant No. 2011AA060403). The research also got support from the opening research funds from Beijing General Research Institute of Mining \&Metallurgy.

\section{REFERENCE}

[1] Gu H S, Jiang Y. The development of underground equipments, Journal of Mining Equipment, 2014,30(7):40 42.

[2] Gao M X. The Development of Technology on Underground Loader and Underground Automobile Automation. Journal of Modern Mining, 2009, 12(2):1-6.

[3] N. Koyachi, S. Sarata, Unmanned loading operation by autonomous wheel loader, in: ICROS-SICE International Joint Conference, Fukuoka International Congress Center, Japan, August 18- 21, 2009.

[4] Marshall J, Barfoot T, Larsson J. Autonomous Underground Tramming for Center-Articulated Vehicles. Journal of Field Robotics, 2008, 25(6-7):400-421.

[5] CHEN M, WANG L, JIA M. An Overview of Autonomous Navigation Techniques and Development Trend for Underground LHD. Journal of China Safety Science Journal, 2013, 23(3):130-134.

[6] CHI H P, ZHAN K, SHI B Q, Automatic guidance of underground mining vehicles using laser sensors. Journal of tunnelling and underground space technology 27(2012), pp.142-148.

[7] Telegrams for Operating/Configuring the LMS 5xx Laser Measurement System (Firmware Version V2.10). SICK AG. Division Auto Ident, Germany. 